\title{
On the Physics of Harmonic Injection in a Traveling Wave Tube
}

\author{
John G. Wöhlbier, Member, IEEE, John H. Booske, Senior Member, IEEE, and Ian Dobson, Senior Member, IEEE
}

\begin{abstract}
The physics of signal injection to shape the output spectrum in a traveling wave tube (TWT) is studied using an analytic solution to the approximate nonlinear S-MUSE model and with the large signal code LATTE. The results verify the long-standing conjecture that a frequency canceled by signal injection is composed of a component due to the injected signal and a component due to the nonlinearity of the TWT. Furthermore, the structures of the solutions are exploited to explain and predict behavior for various signal injection schemes. The scenarios studied include second harmonic injection to reduce the second harmonic and enhance the fundamental, multiple harmonic injection to eliminate the sensitive output power dependence on injected phase, second harmonic injection to reduce intermodulation distortion, and multiple signal injection to reduce the intermodulation spectrum. Insights are given regarding the effectiveness of an injection depending on whether the injected signal is within or outside the linear gain bandwidth of the TWT.
\end{abstract}

Index Terms-Harmonic injection, intermodulation, traveling wave tube (TWT).

\section{INTRODUCTION}

$\mathbf{T}$ RAVELING wave tubes (TWTs) are widely used as amplifiers in communications and electronic countermeasure systems. Due to the nonlinear nature of the device, a signal amplified by a TWT is distorted, compromising the device performance. In a communications application the distortion may manifest as two channels producing energy in a third channel via third-order intermodulation products (3IMs). In this case, the channels must operate such that "cross talk" into other channels is at acceptable levels, which in some applications can be as much as $60 \mathrm{~dB}$ below the fundamental signals [1]. In electronic countermeasure applications, frequency agility is necessary and harmonic generation can limit the usable amplifier bandwidth.

One way to avoid the TWT's nonlinear effects is to reduce the input drive power until the undesired spectral components are at acceptable levels. This method is not an ideal solution because it reduces the overall efficiency of the TWT. Another widely used method for reducing undesired spectral content is

Manuscript received May 23, 2003; revised September 24, 2003. The work of J. G. Wöhlbier and J. H. Booske was supported in part by the U.S. Air Force Office of Scientific Research (AFOSR) under Grant 49620-00-1-0088 and by DUSD (S\&T) under the Innovative Microwave Vacuum Electronics Multidisciplinary University Research Initiative (MURI) program, managed by the USAFOSR under Grant F49620-99-1-0297.

J. G. Wöhlbier is with the Los Alamos National Laboratory, Los Alamos, NM 87545 USA (e-mail: wohlbier@lanl.gov).

J. H. Booske and I. Dobson are with the Department of Electrical and Computer Engineering, University of Wisconsin, Madison, WI 53706 USA (e-mail booske@engr.wisc.edu).

Digital Object Identifier 10.1109/TPS.2004.828820 harmonic injection. Harmonic injection has been studied experimentally [2]-[6] and numerically [6]-[9]. It has been shown that for two drive frequencies, injection of second harmonics of the drive frequencies can reduce the 3IM spectra [3], [5], [9]. Since it is typically the 3IM spectra that is responsible for the "cross talk" between channels, harmonic injection allows one to drive the channel carriers closer to saturation, thus increasing the amplifier efficiency. In electronic countermeasure applications one can inject the second and higher harmonics to extend the usable bandwidth of the amplifier [2], [4].

Mendel [10] claims that harmonic injection is a process of cancellation "... whereby the injected second-harmonic signal is such that it is $180^{\circ}$ out of phase with the second harmonic signal generated by the nonlinear processes inherent in the interaction mechanism." This view is supported by Garrigus and Glick [4] who went as far as providing a speculative drawing of what the waveforms might look like internal to the TWT. However, no quantitative nonlinear theories have been reported that describe the second harmonic signal being made up of an "injected" part and a part "generated by the nonlinear processes inherent in the interaction mechanism." Large signal TWT codes have predicted the phenomenon of canceling the second harmonic with harmonic injection; however, the harmonic in these models was not resolvable into separate components.

In this paper, we develop a general theory of "signal injection" using approximate analytic solutions to the spectral TWT model S-MUSE [11]. The approximate solutions are sums of "injected" terms and terms "generated by the nonlinear processes inherent in the interaction mechanism." Where appropriate, we test our insights from the approximate analytic solution in saturated conditions with the large signal code LATTE [11]. LATTE is derived from the same starting equations as S-MUSE and is therefore ideally suited for this purpose. We study in detail two cases of signal injection. First, harmonic injection to suppress the harmonic and enhance the fundamental is considered. Included in this study is the effect of multiple harmonic injection. Second, we study signal injection to cancel third-order intermodulation products. Harmonic injection, difference frequency injection, 3IM injection, and combinations of these schemes are considered.

In Section II, we develop the signal injection theory. The applications are given in Section III, and the paper is concluded in Section IV. The appendixes provide formulas to compute the analytic solutions.

\section{THEORY}

The S-MUSE model [11] is derived from a one-dimensional (1-D) nonlinear model which uses transmission line equations 
to represent the slow wave circuit and Eulerian electron beam equations. The model is steady state and assumes that all frequencies present are integer multiples of some base frequency $\omega_{0}$. There are five quantities in the TWT description: transmission line voltage $V$, transmission line current $I$, space-charge electric field $E$, electron beam velocity $v$, and electron beam charge density $\rho$. The S-MUSE model is a system of ordinary differential equations for the spatially dependent complex Fourier coefficients of the five quantities $\tilde{V}_{\ell}(z), \tilde{I}_{\ell}(z), \tilde{E}_{\ell}(z)$, $\tilde{v}_{\ell}(z)$, and $\tilde{\rho}_{\ell}(z)$ where $\ell$ is a frequency index and $z$ is the axial distance. Circuit power and voltage phase are denoted as $P_{\ell}(z)$ and $\varphi_{\ell}(z)$; see [11] for details of S-MUSE.

\section{A. Analytic Solution Structure}

S-MUSE can be solved analytically using a series solution [12]. Similar to linear Pierce theory [13], the solution for a state variable at a particular frequency is a sum of complex exponential modes. However, whereas the solution in the linear theory has a finite number of modes, the analytic solution to S-MUSE has an infinite number of modes. We use a finite number of modes to form approximate solutions. Furthermore, when appropriate, we consider only the modes that dominate the solution near the output of the TWT, which are typically those modes with the largest growth rates.

In general, the solution of the voltage at frequency $f_{\ell} \omega_{0}$ may be approximated as

$$
\begin{aligned}
V_{\ell}(z, t)= & \left\{A_{\ell}^{\mathrm{dr}} \exp \left(\mu_{\ell}^{\mathrm{dr}}+i \kappa_{\ell}^{\mathrm{dr}}\right) z\right. \\
& \left.+\sum_{q} A_{\ell}^{\mathrm{nl}[q]} \exp \left(\mu_{\ell}^{\mathrm{nl}[q]}+i \kappa_{\ell}^{\mathrm{nl}[q]}\right) z\right\} \\
& \times e^{i f_{\ell} \omega_{0}\left(\left(z / u_{0}\right)-t\right)}
\end{aligned}
$$

where the superscript dr refers to "driven" quantities and the superscript $\mathrm{nl}$ refers to quantities generated by nonlinear interactions.

The idea of all of the injection schemes is to adjust the amplitudes and phases of the inputs such that the bracketed term in (1) is minimized at $z=L$, where $L$ is the TWT output. This is in contrast to the view that the undesired signal is canceled at all points along the length of the TWT. In the sections of our paper that describe the different injection schemes, we will identify the structure of the different elements of (1). Appendix II provides formulas for computing the quantities appearing in (1).

Depending on the injection scheme, there may be no "driven" portion of (1). For example, in harmonic injection to cancel a 3IM, the naturally generated (nonlinear) 3IM cancels with a mode that is a nonlinear product of the injected harmonic and one of the drive frequencies. In this case, the only dominant modes present are represented in the sum over $q$, i.e., $A_{\ell}^{\mathrm{dr}}=0$. For cases in which the injected harmonic is out of the linear gain bandwidth, the driven portion of (1) may also need to be represented by a sum over modes. To compute the modes in these cases the reader is referred to the general solution in Appendix I.

The solutions to the S-MUSE model of the form (1) are approximations which only apply prior to TWT saturation. By using the large signal code LATTE we will show with simulations that the large signal solutions also have a structure that is composed of modes due to the nonlinearity and modes due to the injected signal.

\section{B. Electron Beam Diagnostics}

The electron beam current modulation is often considered an important quantity in TWT physics. To study harmonics of the electron beam current during harmonic injection, we will need the following quantities. The instantaneous electron beam current in Eulerian coordinates is given by

$$
i_{\text {beam }}=S \rho v
$$

where $S$ is the electron beam cross-sectional area. The Fourier coefficient of this expression at frequency $f_{\ell} \omega_{0}$ is

$$
\left\langle i_{\text {beam }}(z)\right\rangle_{\ell}=S \frac{1}{2 \pi} \int_{2 \pi} \rho v e^{-i f_{\ell} \psi} d \psi .
$$

Substituting the Fourier series for $\rho(z, \psi)$ and $v(z, \psi)$, e.g., $v(z, \psi)=\sum_{\ell=-\infty}^{\infty} \tilde{v}_{\ell}(z) e^{i f_{\ell} \psi}$ into (3), one gets

$$
\left\langle i_{\text {beam }}(z)\right\rangle_{\ell}=S \sum_{\substack{m, n \\ f_{m}+f_{n}=f_{\ell}}} \tilde{\rho}_{m} \tilde{v}_{n}
$$

where $\tilde{\rho}_{m}, \tilde{v}_{n}$ are MUSE variables, i.e., spatially dependent Fourier coefficients, and the sum is over frequency pairs such that the frequencies add up to $f_{\ell} \omega_{0}$.

For the Lagrangian model LATTE the beam current harmonics are given by

$$
\left\langle i_{\text {beam }}(z)\right\rangle_{\ell}=\frac{1}{2 \pi} \int_{2 \pi} I_{0}\left(\psi_{0}\right) e^{-i f_{\ell} \Psi\left(z, \psi_{0}\right)} d \psi_{0}
$$

where $\psi_{0}$ is the Lagrangian "disk" label and $\Psi\left(z, \psi_{0}\right)$ is the function describing the phase of the disk $\psi_{0}$ as a function of axial position. For an unmodulated beam $I_{0}\left(\psi_{0}\right)=I_{0}$.

In Section III, we will compute $\left\langle i_{\text {beam }}(z)\right\rangle_{\ell}$ for different values of $\ell$ using the code LATTE/MUSE Numerical Suite. lmsuite solves the MUSE models and the large signal code LATTE for arbitrary TWT geometries. ${ }^{1}$

\section{APPLICATIONS}

In this section, we provide several examples of the harmonic injection theory. The first example is harmonic injection to suppress the harmonic or enhance the fundamental, and the second example is signal injection to suppress an intermodulation product. ${ }^{2}$ We study various aspects of the physics that the solutions afford.

For the studies, we use TWT parameters based on the X-WING TWT [5]. The electron beam parameters are listed in Table I and the relevant dispersion parameters (cold circuit phase velocity, beam-averaged interaction impedance, and space-charge reduction factor) are listed in Table II. For frequencies not listed in Table II, dispersion parameters are determined by a linear interpolation between neighboring frequencies; see [11] for a full description of how the dispersion parameters enter into the model equations. The TWT length is $L=15 \mathrm{~cm}$. Unless otherwise noted, we do not include a circuit

\footnotetext{
${ }^{1}$ The code and the input files used in this paper are available at http://www.lmsuite.org.

${ }^{2} \mathrm{We}$ define a $K$ th-order intermodulation product of frequencies $f_{1}, f_{2}, \ldots, f_{P}$ as $r_{1} f_{1}+r_{2} f_{2}+\cdots+r_{P} f_{P}$ where $r_{j}$ are integers (possibly zero) and $K=\left|r_{1}\right|+\left|r_{2}\right|+\cdots+\left|r_{P}\right|$. This structure includes fundamental and harmonic frequencies if all but one of the $r_{j}$ are zero.
} 
TABLE I

XWING TWT ELECTRON BEAM AND CiRCUIT PARAMETERS

\begin{tabular}{ll} 
Parameter & Value \\
\hline Cathode voltage & $-2.75 \mathrm{kV}$ \\
Beam current & $0.22 \mathrm{~A}$ \\
Beam radius & $0.55 \mathrm{~mm}$ \\
Helix radius & $1.4 \mathrm{~mm}$ \\
\hline
\end{tabular}

TABLE II

XWING TWT DISPERSION PARAMETERS

\begin{tabular}{cccc}
$f(\mathrm{GHz})$ & $\tilde{v}_{\mathrm{ph}}\left(\times 10^{7} \mathrm{~m} / \mathrm{s}\right)$ & $\tilde{K}(\Omega)$ & $\tilde{R}$ \\
\hline 1.00 & 2.480 & 139.0 & $1.55 \times 10^{-2}$ \\
2.00 & 2.486 & 104.0 & $4.66 \times 10^{-2}$ \\
3.00 & 2.498 & 64.0 & $8.40 \times 10^{-2}$ \\
4.00 & 2.514 & 38.0 & $1.24 \times 10^{-1}$ \\
5.00 & 2.533 & 24.0 & $1.65 \times 10^{-1}$ \\
6.00 & 2.550 & 15.0 & $2.06 \times 10^{-1}$ \\
7.00 & 2.563 & 10.0 & $2.45 \times 10^{-1}$ \\
8.00 & 2.577 & 4.0 & $2.83 \times 10^{-1}$ \\
\hline
\end{tabular}

sever so that we consider only the essential physics of signal injection. We treat separately the cases where the sever plays an important role in the injection physics. The small signal gain as a function of frequency for these parameters as computed by S-MUSE is given in Fig. 1.

Throughout the remainder of the paper, circuit inputs are specified by input power and phase. These values are used to compute input circuit voltage and current amplitudes with (23) and (24) of [11].

\section{A. Fundamental and Harmonic Frequencies}

First, we consider second harmonic injection in the presence of one drive frequency. We study the effect of the injected second harmonic on the fundamental and harmonic frequencies in two cases. In the first case, the harmonic signal is in the linear gain bandwidth, and in the second case the harmonic is out of the linear gain bandwidth. ${ }^{3}$ We then consider injecting the second and third harmonic to cancel the second harmonic.

1) Harmonic in Linear Gain Bandwidth: In the first example, we use the fundamental input frequency of $2 \mathrm{GHz}$ and the injected second harmonic at $4 \mathrm{GHz}$. The voltage solution (1) for each of these frequencies has a driven mode and a nonlinear mode. There is a nonlinear mode at $2 \mathrm{GHz}$ because $2 \mathrm{GHz}$ is the difference frequency of 4 and $2 \mathrm{GHz}$. The nonlinear mode at $4 \mathrm{GHz}$ is the second harmonic of $2 \mathrm{GHz}$. In Fig. 2, we show solutions of (1) for this case. Fig. 2(a) shows that the output power of the fundamental is maximized for an optimum input harmonic phase. At $2 \mathrm{GHz}$, the nonlinear mode of (1) increases monotonically with increasing harmonic input power at fixed phase, and therefore the power output at the fundamental increases monotonically for increasing harmonic input power as seen in Fig. 2(a). The power enhancement at the fundamental when the harmonic and fundamental have equal input powers $(5 \mathrm{dBm})$ is $1 \mathrm{~dB}$ for optimum phase.

${ }^{3}$ We define a frequency to be in the linear gain bandwidth if it has an exponentially growing solution predicted by the linear theory [13]. For the parameters provided in Tables I and II, the frequencies between 1 and $6 \mathrm{GHz}$ are in the linear gain bandwidth.

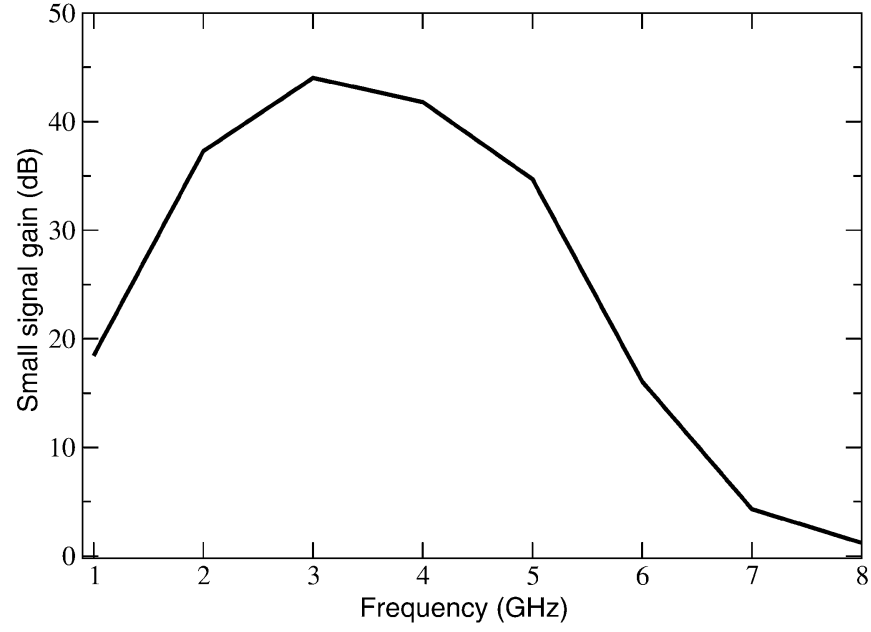

Fig. 1. Small signal gain of XWING TWT parameters as a function of frequency. Curve was computed with the S-MUSE model.

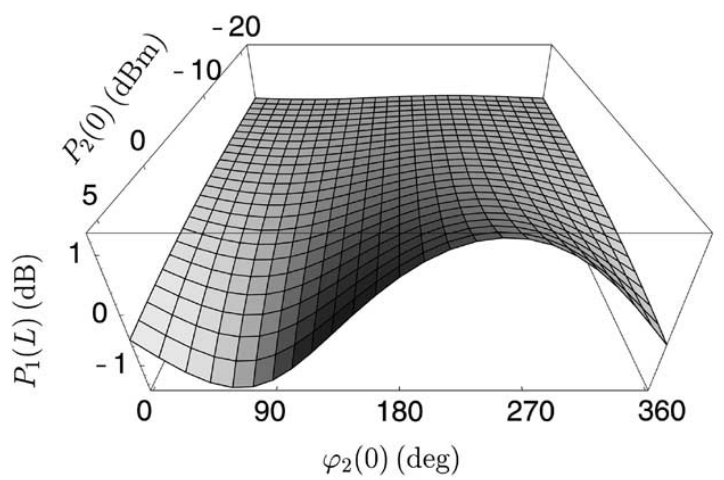

(a) Output power at $2 \mathrm{GHz}$ as a function of injected harmonic power and phase. The optimum second harmonic input phase is $\varphi_{2}(0)=244^{\circ}$. At this input phase the fundamental output power increases monotonically with increasing second harmonic input power until saturation effects set in.

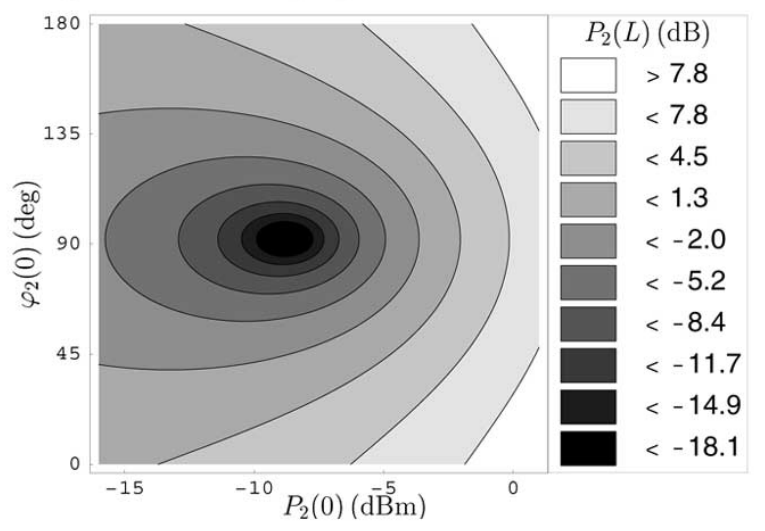

(b) Output power at $4 \mathrm{GHz}$ as a function of injected harmonic power and phase $P_{2}(L)$ is in dB with respect to output power at $4 \mathrm{GHz}$ with no harmonic injection The optimum cancellation harmonic inputs are $P_{2}(0)=-8.86 \mathrm{dBm}, \varphi_{2}(0)=$ $92.25^{\circ}$.

Fig. 2. Output power at (a) fundamental (2 GHz) and (b) second harmonic (4 $\mathrm{GHz})$ as a function of injected harmonic power $P_{2}(0)$ and injected harmonic phase $\varphi_{2}(0)$ predicted by (1) for second harmonic injection. For both figures, the fundamental input power and phase are $P_{1}(0)=5 \mathrm{dBm}, \varphi_{1}(0)=0.0^{\circ}$. With no harmonic injection the harmonic is $9.23 \mathrm{~dB}$ below the fundamental at the TWT output.

Fig. 2(b) shows that for injected second harmonic powers between -10 and $-8 \mathrm{dBm}$ and phases between $85^{\circ}$ and $95^{\circ}$ the harmonic is suppressed by more than $18 \mathrm{~dB}$. The center of 


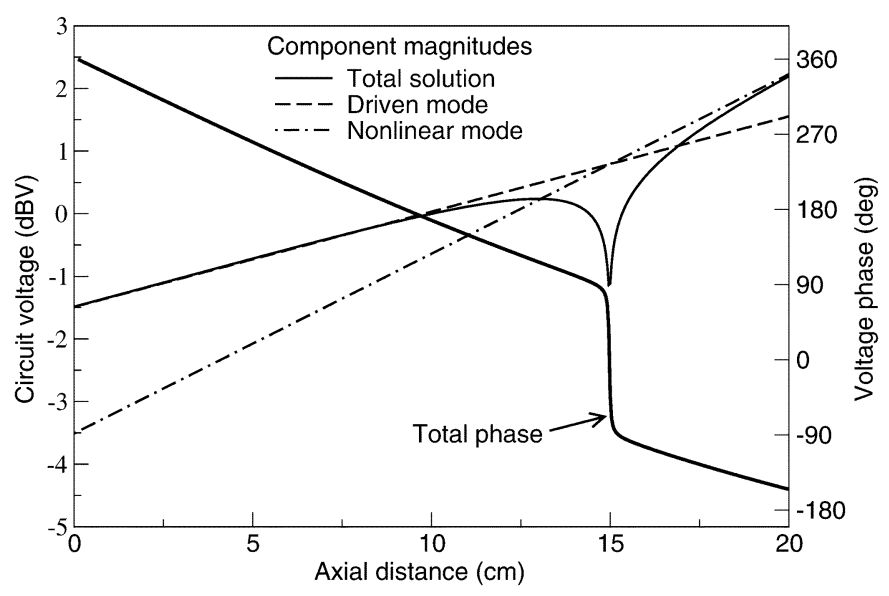

Fig. 3. Magnitude and phase of (1) and component magnitudes of (1) for second harmonic $4.0 \mathrm{GHz}$ with second harmonic injection to achieve second harmonic cancellation. Driven mode dominates the solution prior to $z=15 \mathrm{~cm}$, and the nonlinear mode dominates the solution after $z=15 \mathrm{~cm}$. This can be seen from the component magnitudes as well as the $180^{\circ}$ phase change of the total solution at $z=15 \mathrm{~cm}$. Fundamental and second harmonic input powers and phases are 5.0 and $-8.86 \mathrm{dBm}$ and $0.0^{\circ}$ and $92.25^{\circ}$, respectively. Voltage phase is with respect to the cold circuit wave at $4 \mathrm{GHz}$.

this range corresponds to total cancellation of the second harmonic signal $(-\infty \mathrm{dB})$. Experimental realization of this theoretical perfect cancellation is not possible. Line-broadening effects such as phase jitter, shot noise, and voltage supply noise will leave a small nonzero amplitude at the point of optimal suppression. For a case similar to Fig. 2(b), Singh et al. have experimentally realized harmonic suppression of approximately $30 \mathrm{~dB}$ [6]. With improved instrumentation it is likely that the amount of suppression could be increased.

From Fig. 2(b) and (1), we see that the optimum injected harmonic power and phase are independent of each other. For any injected harmonic power a scan of injected harmonic phase may be made to determine the optimum phase. With the injected harmonic phase set to this optimum value the harmonic injected power may be scanned to determine the optimum cancellation point. This method of detecting maximum cancellation has been confirmed experimentally for nonsaturated operation [5].

In Fig. 3, we show the modes of (1) that make up the harmonic solution for the optimum cancellation inputs of Fig. 2(b). Shown are the magnitudes of the separate modes and the magnitude and phase of the total solution (1). The figure clearly shows that the axial position of harmonic cancellation (the TWT output) is the point at which the dominant mode of the solution changes from the driven mode to the nonlinear mode. The $180^{\circ}$ phase change in the composite solution also illustrates this point.

To test whether the qualitative conclusions about the structure of the solutions during harmonic injection hold more generally, we next consider results of LATTE simulations for input powers that produce power saturation at the fundamental frequency. We find that for saturated operation a single global minimum of harmonic output power versus input power and phase [see Fig. 2(b)] ceases to exist. Rather, several local minima form. To determine the global minimum requires an extensive and highly resolved scan over input power and phase. In Fig. 4, we show the second harmonic output power as a function of injected second harmonic input power and

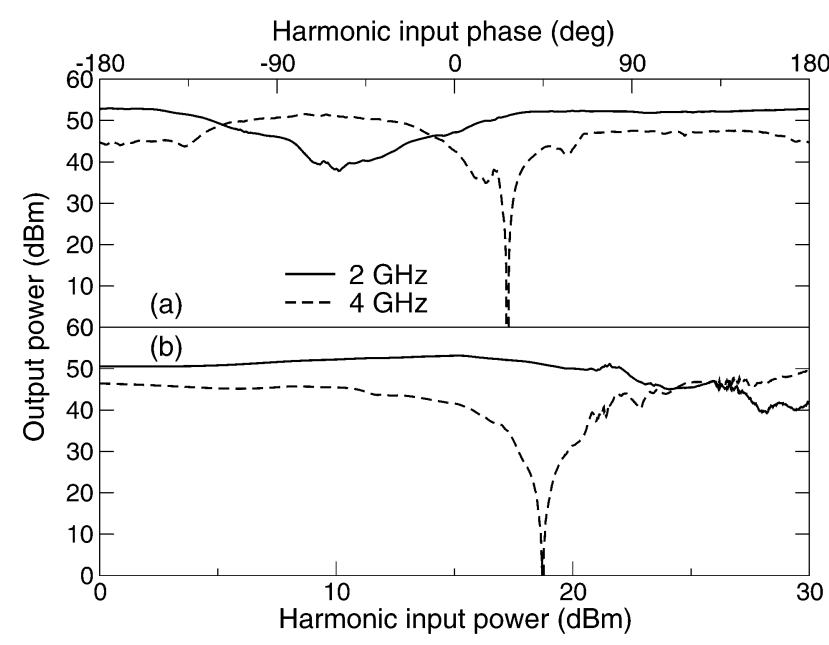

Fig. 4. Fundamental and harmonic output power versus (a) harmonic input phase and (b) harmonic input power predicted by LATTE. Fundamental input power produces saturated output with no harmonic injection. Fundamental input power and phase are $20.0 \mathrm{dBm}$ and $0.0^{\circ}$, respectively, for (a) and (b). In (a), harmonic input power is $18.75 \mathrm{dBm}$, and in (b) harmonic input phase is $26.475^{\circ}$.

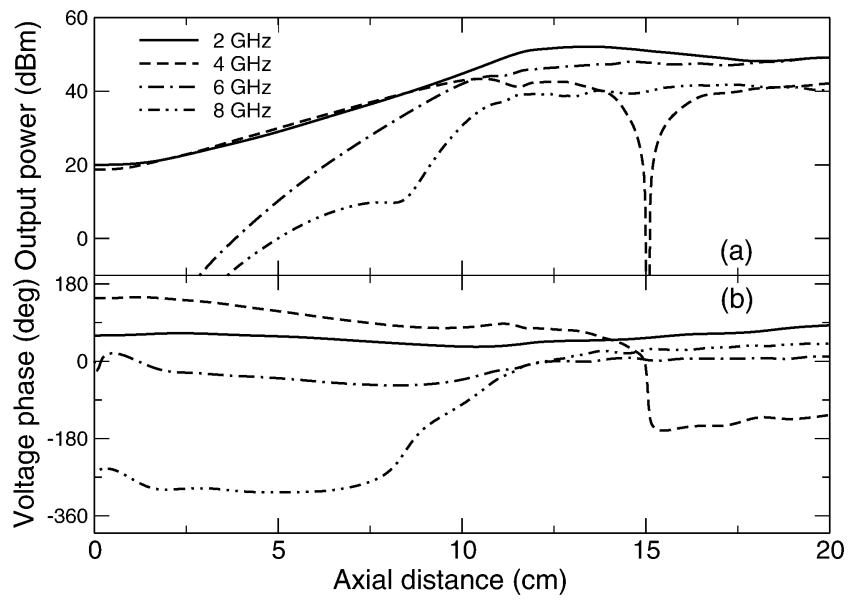

Fig. 5. (a) Output power and (b) voltage phase for fundamental through fourth harmonic predicted by LATTE, with second harmonic injection to cancel the second harmonic. Abrupt phase change of $180^{\circ}$ in the second harmonic is evidence that even for saturated operation the second harmonic solution is comprised of two modes as in the approximate analytic solution (1). Fundamental input power and phase of $20.0 \mathrm{dBm}$ and $0.0^{\circ}$ produce saturation at $z=15 \mathrm{~cm}$ in absence of harmonic injection. Second harmonic input power and phase are $18.75 \mathrm{dBm}$ and $26.48^{\circ}$, respectively. Voltage phases are with respect to the cold circuit waves at the respective frequencies.

phase for a fundamental input power that drives the TWT into saturation. The simulations for saturated operation include the fundamental through fourth harmonic spectral components in the circuit wave and electron beam.

To show that the second harmonic voltage in the TWT has the structure of (1) even for saturated operation, we can consider the phase of the voltage predicted by LATTE simulations. As in Fig. 3, we expect to see the driven mode character dominate prior to the point of cancellation and the nonlinearly generated mode character after cancellation. If this is true in the saturated case, then the voltage phase of the harmonic should jump by $180^{\circ}$ at the point of cancellation. In Fig. 5, we show output powers and voltage phases for the fundamental through fourth harmonic with second harmonic injection to cancel the second 


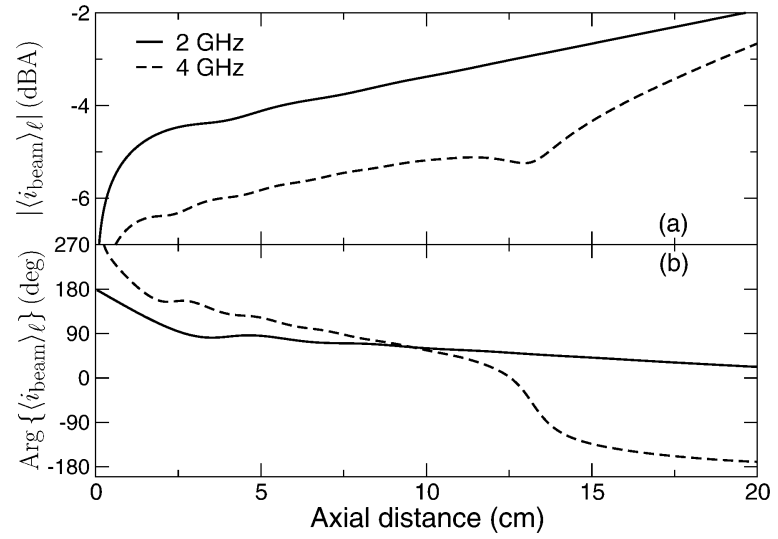

Fig. 6. (a) Beam current modulation magnitude and (b) phase at fundamental and second harmonic for second harmonic injection to cancel the second harmonic at $z=15 \mathrm{~cm}$ predicted by LATTE. Harmonic beam current modulation "changes modes" at about $z=13 \mathrm{~cm}$, as evidenced by magnitude dip and phase change. However, the modes do not cancel to produce zero beam current second harmonic modulation $(-\infty \mathrm{dB})$ at any point along the TWT. Fundamental and second harmonic input power and phase are -20.0 and $-57.5 \mathrm{dBm}$ and $0.0^{\circ}$ and $91.0^{\circ}$, respectively, well below powers which produce saturation effects. Beam current modulation magnitudes are in decibels with respect to $1 \mathrm{~A}$, and beam current modulation phases are with respect to cold circuit waves at the respective frequencies.

harmonic at $z=15 \mathrm{~cm}$. The fundamental input power is such that without harmonic injection the output power at the fundamental is saturated. With harmonic injection, the fundamental output power at the output $(z=15 \mathrm{~cm})$ is compressed by about $1 \mathrm{~dB}$ as the saturation point has shifted to $z=13.6 \mathrm{~cm}$. One sees that the output phase at the second harmonic abruptly changes by $180^{\circ}$ at the cancellation point. This matches the structure of the two-mode analytic solution of the S-MUSE model.

For the same input frequencies, we next consider electron beam current modulation magnitude and phase at the fundamental and second harmonic. Equation (4) gives the current modulation at frequency $f_{\ell} \omega_{0}$ in MUSE variables. The beam charge density $\tilde{\rho}_{m}$ and beam velocity $\tilde{v}_{n}$ have the form of (1), where the exponents $\left(\mu_{m, n}^{\mathrm{dr}}+i \kappa_{m, n}^{\mathrm{dr}}\right.$ and $\left.\mu_{m, n}^{\mathrm{nl}[q]}+i \kappa_{m, n}^{\mathrm{nl}[q]}\right)$ will be the same as those of the voltage, but the complex amplitudes ( $A_{m, n}^{\mathrm{dr}}$ and $A_{m, n}^{\mathrm{nl}[q]}$ ) will be different. For the case of second harmonic injection with a single fundamental input, the solutions for the charge density and beam velocity at the fundamental $\tilde{\rho}_{1}$ and $\tilde{v}_{1}$ are composed of a driven mode and a nonlinearly generated mode. Therefore, the beam current modulation at the second harmonic $\left\langle i_{\text {beam }}(z)\right\rangle_{2}$, i.e., the product of $\tilde{\rho}_{1}$ and $\tilde{v}_{1}$, may be written as a sum of three terms (the cross terms have the same exponents and may be combined). There is no guarantee that since the inputs are set such that the circuit voltage modes cancel at the TWT output, the modes making up the current modulation will also cancel. In fact, in all of the cases that we have studied, we have found that the current modulation is not canceled for any point along the length of the TWT. However, the theory predicts that such a cancellation of the beam modulation modes is possible for the proper inputs.

In Fig. 6, we show the beam current modulation magnitude and phase of the fundamental and second harmonic components during harmonic injection to cancel the harmonic voltage at $z=$ $15 \mathrm{~cm}$ as predicted by LATTE. The fundamental input power is small enough so that no saturation effects are encountered

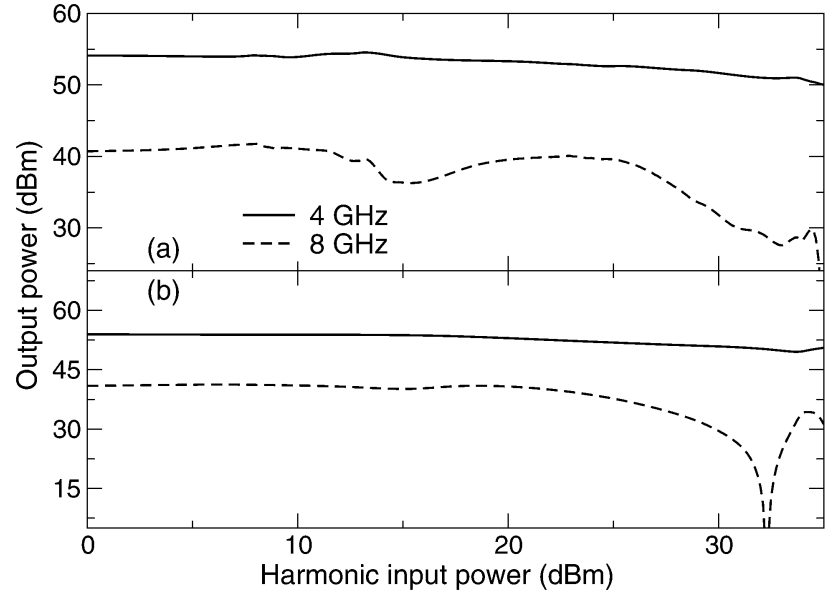

Fig. 7. Fundamental and second harmonic output power versus second harmonic input power for harmonic input phase equal to (a) $47.9^{\circ}$ and (b) $12.5^{\circ}$ predicted by LATTE. In (a), harmonic input phase is set to minimize output harmonic for injected harmonic power of $15 \mathrm{dBm}$, and in (b) harmonic input phase is set to cancel output harmonic for injected harmonic power of $32.29 \mathrm{dBm}$. Fundamental input power and phase of $13.0 \mathrm{dBm}$ and $0.0^{\circ}$ produce saturated output power of $54.1 \mathrm{dBm}$ at the fundamental and $4.06 \mathrm{dBm}$ at the harmonic with no harmonic injection.

in the simulation region. The second harmonic beam current modulation "changes modes" at about $z=13 \mathrm{~cm}$ as evidenced by the dip in the magnitude and the phase change. However, this is well before the voltage cancellation which occurs at $z=$ $15 \mathrm{~cm}$. The relationship between the changing of modes in the beam current and the changing of modes in the circuit voltage is a subject of future study.

It has been suggested that harmonic injection might be viewed as input waveform "shaping" such that the beam current waveform is sinusoidal at the output [14]. Fig. 6 indicates that such a view is incorrect, since the harmonic component of the beam current is a large fraction of the fundamental component of the beam current at $z=15 \mathrm{~cm}$, where the second harmonic circuit voltage is canceled.

2) Harmonic Out of Linear Gain Bandwidth: When the second harmonic is out of the linear gain bandwidth, the component of the analytic solution (1) corresponding to the injected harmonic does not have an exponentially growing form, hence does not grow to a level required to cancel the nonlinearly generated harmonic at the output. Thus, in most cases, modest injected harmonic powers will have a small effect on reducing the second harmonic. To get cancellation of the second harmonic, a large value of injected harmonic power is required. For the analytic solution (1) to be a good approximation, additional modes from the full solution in Appendix I should be included to describe the harmonic.

Using LATTE we consider one case of second harmonic injection with the fundamental frequency at $4 \mathrm{GHz}$ and the second harmonic at $8 \mathrm{GHz}$, where the fundamental input is set to achieve saturation at $z=15 \mathrm{~cm}$. In Fig. 7, we plot fundamental and second harmonic output power versus harmonic input power for two different values of fixed second harmonic input phase. In Fig. 7(a), the harmonic input phase is set so that the harmonic output power is minimized for a harmonic input power of $15 \mathrm{dBm}$. For this injection $(15 \mathrm{dBm}$, $47.9^{\circ}$ ), the amount of output harmonic reduction from its 
value of $4.06 \mathrm{dBm}$ with no harmonic injection is about $4 \mathrm{~dB}$. On the other hand, in Fig. 7(b) one can see that cancellation of the second harmonic is obtainable in principle. However, the required injected harmonic power to do so is about $1 \%$ of the fundamental output power. A comparison of Fig. 7 to Fig. 4, and their relative harmonic to fundamental input powers to obtain cancellation $\left(P_{2 f_{1}}-P_{f_{1}}=19.29 \mathrm{~dB}\right.$ and $P_{2 f_{1}}-P_{f_{1}}=-1.25 \mathrm{~dB}$ respectively), illustrates the substantial difference between the case when the second harmonic is in the linear gain bandwidth and when the second harmonic is out of the linear gain bandwidth.

Almost all TWTs have circuit severs to control internal reflections. We expect that the presence of the sever will highly attenuate, if not totally eliminate, the nongrowing injected harmonic mode. Since the injected mode is the mode that allows for reduction or cancellation of the second harmonic, we expect that when a sever is included, reduction of the second harmonic should be possible but a total cancellation is likely not possible for reasonable harmonic injection powers. For LATTE simulations including a sever where we restricted the harmonic input to less than $10 \%$ of the fundamental output, the maximum amount of harmonic suppression we obtained was $16.4 \mathrm{~dB}$, from 38.8 to $22.4 \mathrm{dBm}$, for a fundamental input of $27.0 \mathrm{dBm}$ and a harmonic input of $34.5 \mathrm{dBm}$. The sever is modeled by a resistive loss in the center section of the circuit. For details on how the sever is incorporated into the circuit equations, see [12, Appendix A]. For details of the loss profile one can run the lmsuite input decks. ${ }^{4}$

3) Second and Third Harmonic Injection: Next, we consider injection of the second and the third harmonic to achieve cancellation of the second harmonic when the second and third harmonics are both in the linear gain bandwidth. For this injection scheme there are three terms in (1) for each of the three frequencies: one driven term and two nonlinear terms. To achieve cancellation for a particular frequency at the output, these three terms should add to zero at $z=L$.

As an example, we solve the S-MUSE equations for a fundamental frequency of $1.5 \mathrm{GHz}$, second harmonic of $3.0 \mathrm{GHz}$, and third harmonic of $4.5 \mathrm{GHz}$. The respective input powers and phases for the fundamental, second, and third harmonics are $10,-14.2$, and $-10.6 \mathrm{dBm}$, and $0.0^{\circ}, 15.0^{\circ}$, and $-45.0^{\circ}$. When evaluated at $z=L$, the three terms in (1) for the second harmonic frequency may be represented in a phasor diagram as seen in Fig. 8. The phasor $\mathbf{A}$ represents the nonlinear product of the fundamental with itself (the mode we wish to cancel), $\mathbf{B}$ represents the driven second harmonic, and $\mathbf{C}$ represents the nonlinear product of the third harmonic with the fundamental. If a phasor $\mathbf{X}$ has magnitude $|\mathbf{X}|$ and phase $\theta_{\mathbf{X}}=\operatorname{Arg}\{\mathbf{X}\}$, then note from Fig. 8 that for cancellation $|\mathbf{A}|=|\mathbf{B}+\mathbf{C}|$ and that $\theta_{\mathbf{B}+\mathbf{C}}-\theta_{\mathbf{A}}=\pi$.

For sufficiently small fixed fundamental input power and fixed fundamental, second, and third harmonic input phases, if $0<\left|\theta_{\mathbf{C}}-\theta_{\mathbf{B}}\right|<\pi$ then the second and third harmonic input powers can be adjusted to achieve second harmonic cancellation. This replaces the necessity of having precise control of the input phase of the injected harmonic in the case of single harmonic injection with the necessity of having precise control

${ }^{4}$ Found at http://www.lmsuite.org.

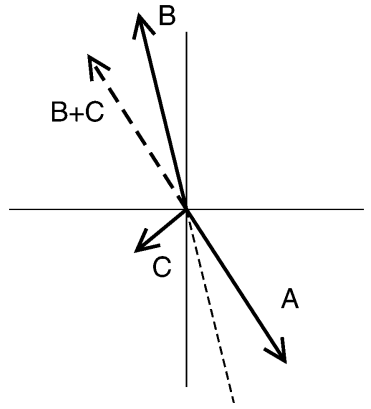

Fig. 8. Output phasor picture produced by analytic S-MUSE solution for second and third harmonic injection. Phasor $\mathbf{A}$ represents the second harmonic mode due to nonlinear product of fundamental with itself, phasor $\mathbf{B}$ represents the injected second harmonic mode, and phasor $\mathbf{C}$ represents the mode due to the nonlinear product of the third harmonic with the fundamental. Phasor $\mathbf{B}+\mathbf{C}$ cancels phasor $\mathbf{A}$

of the second and third harmonic input powers. In hardware it is typically easier to precisely control input powers than phases. By "sufficiently small input power" we mean, for example, that when only the fundamental is injected that neither the fundamental nor the nonlinearly generated second and third harmonic show signs of onset of saturation at $z=L$. In fact, the principle being described may hold for larger input powers, but we have not tried to determine an upper limit on the input powers for which it holds.

Using LATTE we found that for input phases identical to the values used to generate Fig. 8, and respective input powers for the fundamental, second, and third harmonics of 10, -13.3 , and $-9.6 \mathrm{dBm}$, the second harmonic is canceled at $z=15 \mathrm{~cm}$.

From the phasor diagram in Fig. 8, one can see that the relative amounts of injected second and third harmonic power (proportional to the lengths of output phasors $\mathbf{B}$ and $\mathbf{C}$ ) depend on the relative choice of the input phases of the three injected signals. Judicious choice of the input phases may be used to minimize the amount of injected power. In fact, the minimal injected powers would correspond to inputs such that both phasors $\mathbf{B}$ and $\mathbf{C}$ were $180^{\circ}$ out of phase with respect to phasor $\mathbf{A}$, and the lengths of phasors $\mathbf{B}$ and $\mathbf{C}$ were split up such that their magnitudes add to the magnitude of phasor $\mathbf{A}$. However, this situation does require precise phase control on the input.

As in the case of second harmonic injection, one expects that the voltage phase of the solution of the second harmonic at a position $z$ will correspond to the mode which dominates the solution at that position. For inputs below those which produce saturation, the dominant modes are growing exponentially and the $180^{\circ}$ degree phase change in the solution is clearly seen (e.g., Fig. 3). For larger inputs the modes are no longer exponentially growing after the point of cancellation, so the abrupt change in phase may not be as evident in the solution. In Fig. 9, we show the voltage phase of the second harmonic as a function of axial distance along the TWT for the fundamental input powers of 10.0, 23.0, and $28.0 \mathrm{dBm}$, corresponding to linear behavior, 3-dB compression, and full saturation, respectively, as predicted by LATTE. In each case, the input phases for the fundamental, second, and third harmonic are $0.0^{\circ}, 15.0^{\circ},-45.0^{\circ}$ and the second and third harmonic input powers have been set to cancel the second harmonic at $z=15 \mathrm{~cm}$. For linear behavior 


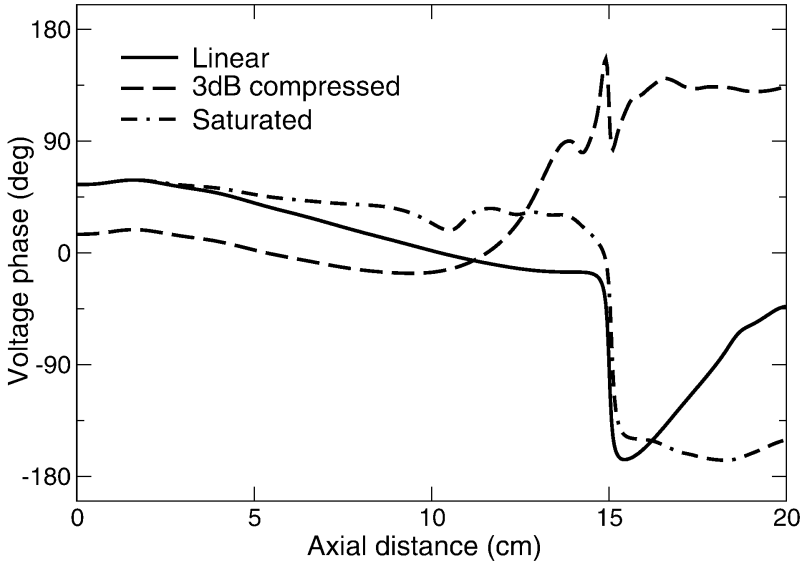

Fig. 9. Voltage phase of the second harmonic with second and third harmonic injection for fundamental input powers in the linear regime, output 3-dB compressed, and output saturated as predicted by LATTE. Phase is with respect to cold circuit phase velocity at $3.0 \mathrm{GHz}$. Second harmonic is canceled at $z=15 \mathrm{~cm}$. All traces show change in phase at cancellation point, but characters are different due to different relative inputs. Fundamental, second, and third harmonic power and phase inputs are: linear 10.0, $-13.3,-9.6 \mathrm{dBm}$, $20.0^{\circ}, 55.0^{\circ}, 15.0^{\circ} ; 3-\mathrm{dB}$ compressed $23.0,11.086,-1.25 \mathrm{dBm}, 0.0^{\circ} 15.0^{\circ}$, $-130.0^{\circ}$; saturated $28.0,12.345,12.55 \mathrm{dBm}, 40.0^{\circ}, 55.0^{\circ}, 270.0^{\circ}$.

and saturation the $180^{\circ}$ phase change is clearly seen, while for $3-\mathrm{dB}$ compression a phase change is still evident, but not as distinct.

Finally, we provide a conjecture about the effect of injecting harmonics higher than third order on canceling an undesired harmonic. The properly phased injection of each additional higher order harmonic results in one more output phasor (see Fig. 8) that can be used to cancel the undesired phasor. This could result in reducing the power required for each injected signal. However, when the injected harmonics are out of the linear gain bandwidth, their resulting phasors may be too small to have an appreciable affect on canceling the undesired harmonic.

\section{B. Intermodulation Frequencies}

Signal injection can also be used to suppress intermodulation frequencies [3], [5], [9]. Consider two drive frequencies $f_{a}, f_{b}$ with $f_{a}<f_{b}$, the second-order products $2 f_{a}, f_{a}+f_{b}$, $2 f_{b}, f_{b}-f_{a}$, and the third-order intermodulation frequencies $2 f_{a}-f_{b}, 2 f_{b}-f_{a}$. Then, for example, injection of the second harmonic $2 f_{a}$ forms a nonlinear second-order product with the fundamental $f_{b}$ which may be adjusted to cancel the nonlinear third-order intermodulation frequency $2 f_{a}-f_{b}$. Similarly, one can inject $2 f_{b}$ to cancel $2 f_{b}-f_{a}$. Furthermore, in principle, one can inject the difference frequencies and form second-order products with the fundamental frequencies to cancel the 3IM frequencies, which has been shown experimentally in solid-state amplifiers [15]. Lastly, one can inject the 3IM frequencies directly with the right amplitudes and phases to achieve cancellation [16]. There are other third-order intermodulation frequencies that we do not list since they are located near the third harmonics and may be removed by filtering.

Similar to the case of multiple harmonic injection, one can use two or more of the above injection frequencies simultaneously to eliminate the need for precise input phase control. The

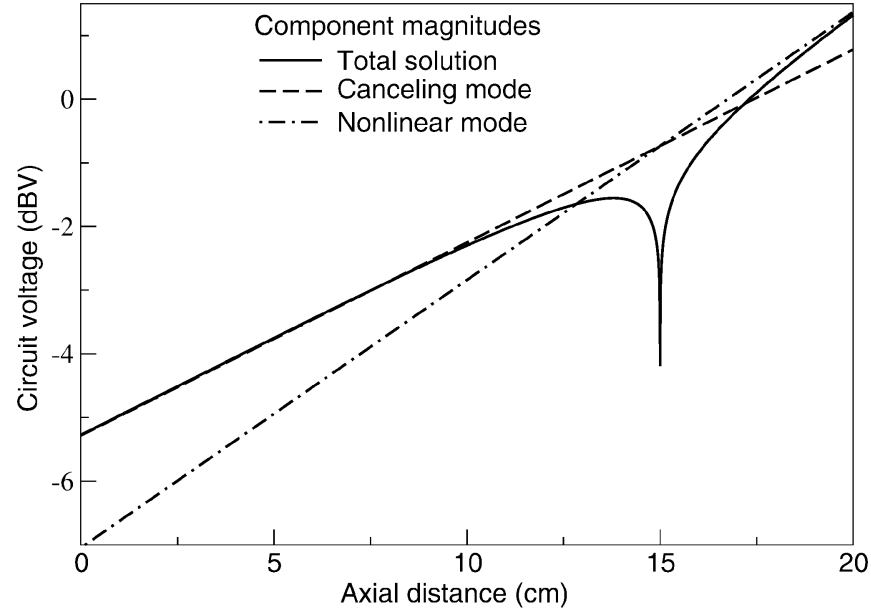

Fig. 10. Magnitude of (1) and component magnitudes of (1) for 3IM frequency $1.8 \mathrm{GHz}$ with second harmonic injection to cancel the 3IM frequency. Canceling mode (nonlinear difference product of $3.8 \mathrm{GHz}$ and $2.0 \mathrm{GHz}$ ) dominates the solution prior to $z=15 \mathrm{~cm}$, and the nonlinear mode dominates the solution after $z=15 \mathrm{~cm}$. Fundamental $(1.9,2.0 \mathrm{GHz})$ and second harmonic $(3.8 \mathrm{GHz})$ input powers and phases are $0.0,0.0,-18.32 \mathrm{dBm}$ and $0.0^{\circ}, 30.0^{\circ}, 116.24^{\circ}$, respectively.

effect of the different injection schemes on the intermodulation frequencies other than those that are being canceled is a subject of future work.

For the examples of Sections III-B1 and 4, we choose the drive frequencies to be 1.9 and $2.0 \mathrm{GHz}$ with input powers of $0.0 \mathrm{dBm}$ and respective input phases of $0.0^{\circ}$ and $30.0^{\circ}$. For the examples of Sections III-B2 and 3, we choose the drive frequencies to be 3.9 and $4.0 \mathrm{GHz}$ with input powers of $-5.0 \mathrm{dBm}$ and respective input phases of $0.0^{\circ}$ and $30.0^{\circ}$. These inputs do not produce saturation effects in any of the frequencies at the TWT output. The behavior of the examples considered here with inputs which produce saturation is a subject of future work. In all of the LATTE simulations, we have accounted for up to the third-order products of the input frequencies. In general, signal injection will result in additional intermodulation frequencies beyond those which exist in the presence of the fundamental frequencies alone.

1) Injection in Linear Gain Bandwidth: We consider first single signal injection when the injected signal is in the linear gain bandwidth. For second harmonic or difference frequency injection, the form of the solution at the third-order intermodulation frequency is given by (1) with $A_{\ell}^{\mathrm{dr}}=0$ and two terms in the sum over $q$. For 3IM injection $A_{\ell}^{\mathrm{dr}} \neq 0$, and there is one term in the sum over $q$. Formulas for $A_{\ell}^{\mathrm{dr}}$ and $A_{\ell}^{\mathrm{n} l[q]}$ are given in Appendix II.

In Fig. 10, we show the mode amplitudes and composite solution envelope from (1) for cancellation with second harmonic injection, and in Fig. 11 we show the mode amplitudes and composite solution for cancellation with 3IM injection. Notice that the induced mode which accomplishes the cancellation in the second harmonic injection case is growing much faster than the driven mode which accomplishes the cancellation in the 3IM injection case (the ranges on the vertical axes of Figs. 10 and 11 are identical). This is because in Fig. 10 the canceling mode is a second-order mode and has a growth rate equal to the sum of the growth rates of the driving frequencies $(2.0$ and $3.8 \mathrm{GHz})$ [17]. 


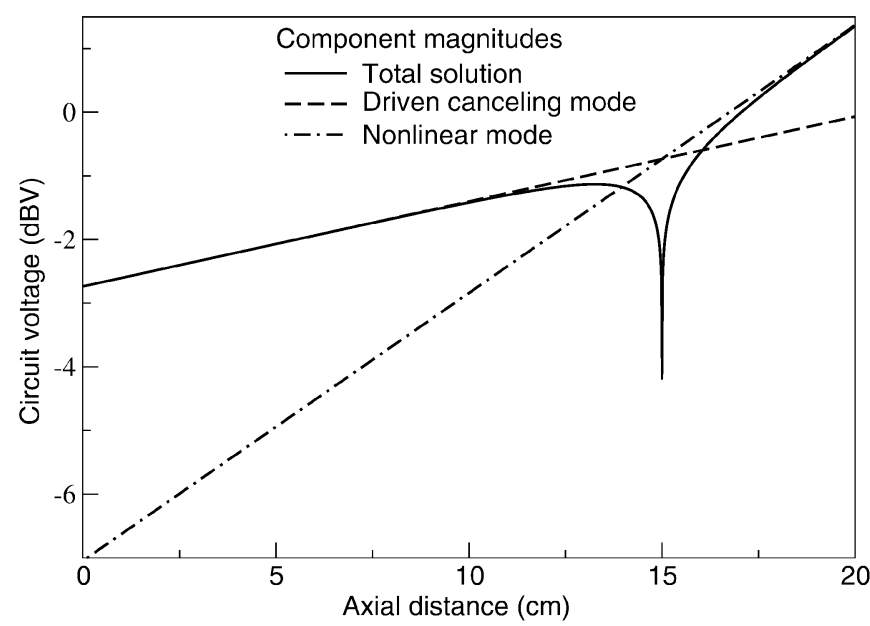

Fig. 11. Magnitude of (1) and component magnitudes of (1) for 3IM frequency $1.8 \mathrm{GHz}$ with $3 \mathrm{IM}$ injection to cancel the $3 \mathrm{IM}$ frequency. Driven mode dominates the solution prior to $z=15 \mathrm{~cm}$, and the nonlinear mode dominates the solution after $z=15 \mathrm{~cm}$. Fundamental $(1.9,2.0 \mathrm{GHz})$ and 3IM $(1.8 \mathrm{GHz})$ input powers and phases are $0.0,0.0,-36.36 \mathrm{dBm}$ and $0.0^{\circ}, 30.0^{\circ}$, $-146.56^{\circ}$, respectively.

In Fig. 11, the canceling mode is a drive frequency and grows with its linear growth rate.

A subtlety in the case of 3IM injection is that with such an injected signal, (1) for the 3IM frequency will have more terms in the sum over $q$. For example, if $f_{3 \mathrm{IM}}$ is the injected 3IM and $f_{a}, f_{b}$ are the original fundamentals, then $f_{3 \mathrm{IM}}$ is equal to the third-order products $\left(f_{3 \mathrm{IM}}+f_{a}\right)-f_{a}$ and $\left(f_{3 \mathrm{IM}}+f_{b}\right)-f_{b}$ (the parentheses $(\cdot)$ indicate the formation of a second-order product, and the subtraction forms a third-order product). That is, the third-order nonlinear solution is now the sum of the original 3IM mode (e.g., $2 f_{a}-f_{b}$ ) in addition to the modes produced by the above third-order products. However, since the required injected power of the 3IM is far smaller than the injected fundamental powers, it was found that the solution was only slightly modified by the inclusion of these additional modes.

2) Injection Out of Linear Gain Bandwidth With No Sever: Similarly to Section III-A-II, we consider the implications of the injected signals being out of the linear gain bandwidth. For narrow band TWTs it is likely that the second harmonic is out of the linear gain bandwidth. For difference frequency injection the difference frequency would be out of the linear gain bandwidth for most drive frequency spacings; however, for wideband TWTs one could construct pairs of input frequencies such that the difference frequency is in the linear gain bandwidth.

We showed in Section III-A2 that second harmonic injection to cancel the second harmonic is much less effective when the second harmonic is out of the linear gain bandwidth. This raises the question of the effectiveness of second harmonic injection to cancel an intermodulation product when the second harmonic is out of the linear gain bandwidth. In this case, the injected second harmonic exists in the circuit as a nongrowing mode. It combines nonlinearly with one of the drive frequencies (via (9) of Appendix I) resulting in a signal that has a growth rate equal to that of the drive frequency [17]. The growth rate of the 3IM frequency to be canceled in most cases will be on the order of three

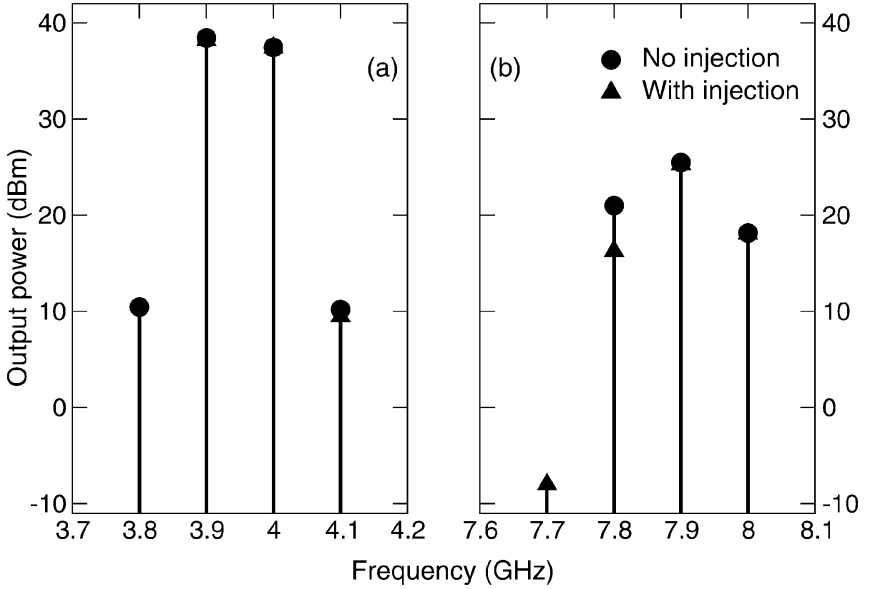

Fig. 12. Output spectrum (a) near fundamentals and (b) near second harmonics with and without second harmonic injection when second harmonic is out of the linear gain bandwidth. Note additional "intermodulation frequencies" (e.g., at $7.7 \mathrm{GHz}$ ) due to injection of the second harmonic. Fundamental inputs 3.9, $4.0 \mathrm{GHz}$ have input power $-5.0 \mathrm{dBm}$ and respective phases of $0.0^{\circ}$ and $30.0^{\circ}$. Injected harmonic $7.8 \mathrm{GHz}$ has input power and phase of $10.6 \mathrm{dBm}$ and $32.2^{\circ}$. Note that there is also partial suppression of the second harmonic at $7.8 \mathrm{GHz}$.

times the growth rate of the fundamental frequency [17]. However, the net levels of the signals can be made comparable since the 3IM starts at a much lower amplitude than the second-order product (the injected second harmonic combined with the fundamental). Therefore, as shown in Fig. 12, harmonic injection to suppress the 3IM when the harmonic is out of the linear gain bandwidth can be effective. In general, the spectra in the remainder of the paper have content near the second, third, and/or fourth harmonics of the drive frequencies, but we have chosen only to display the spectra near the fundamentals and second harmonics.

When the injected second harmonic is out of the linear gain bandwidth the level of the injected signal will generally need to be larger relative to the fundamental input powers as compared to when the injected second harmonic is in the linear gain bandwidth. This can be seen by comparing the values of the injected second harmonic powers relative to the fundamental powers for Fig. $10\left(P_{2 f_{1}}-P_{f_{1}}=-18.32 \mathrm{~dB}\right)$ and Fig. 12 $\left(P_{2 f_{1}}-P_{f_{1}}=15.6 \mathrm{~dB}\right)$. The reason is that when the second harmonic is out of the linear gain bandwidth the second-order mode used to cancel the 3IM grows at a rate about equal to the drive frequency, whereas when the harmonic is in the gain bandwidth the second-order mode used to cancel the 3IM grows at a rate about twice that of the drive frequency [17]. Therefore, to ultimately obtain the same relative power level in the canceling mode, the injected power of the mode with the lower growth rate must be relatively larger. That is, our theory and LATTE simulations indicate that when the second harmonic is out of the linear gain bandwidth, one needs a larger relative harmonic input.

3) Injection Out of Linear Gain Bandwidth With Sever: In contrast to harmonic injection to control the second harmonic when the injected harmonic is out of the linear gain bandwidth as in Section III-A2, we expect from the theory that including a circuit sever will not greatly affect the performance of the injection schemes to control the intermodulation spectrum. The reason is that the mode that cancels the $3 \mathrm{IM}$ is an exponen- 


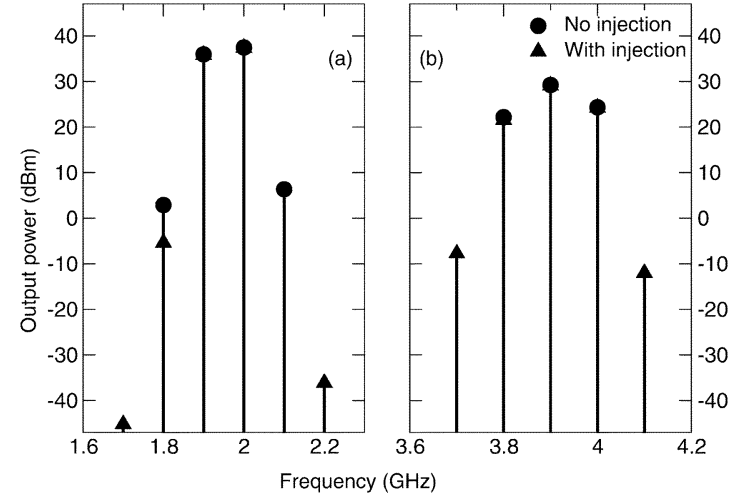

Fig. 13. Output spectrum (a) near fundamentals and (b) near second harmonics with and without difference frequency injection. Note additional "intermodulation frequencies" due to injection of the difference frequency. Fundamental inputs $1.9,2.0 \mathrm{GHz}$ have input power $0.0 \mathrm{dBm}$ and respective phases of $0.0^{\circ}$ and $30.0^{\circ}$. Injected difference frequency $100.0 \mathrm{MHz}$ has input power and phase of $17.4 \mathrm{dBm}$ and $134.45^{\circ}$.

tially growing mode that has a corresponding modulation in the beam current. When the circuit field of this mode is damped by the sever, the beam modulation reinitiates the circuit field mode after the sever. This is in contrast to the case in Section III-A2 where the sever heavily damped the mode that performed the harmonic reduction. In fact, in a LATTE simulation including a circuit sever with fundamental inputs such that the C3IM ratio (the relative level in decibels of the fundamental to the 3IM at the TWT output) without second harmonic injection was the same as those for the simulation that produced Fig. 12, we found that the required harmonic injection power relative to the fundamental power was $P_{2 f_{1}}-P_{f_{1}}=10.5 \mathrm{~dB}$ as compared to $P_{2 f_{1}}-P_{f_{1}}=15.6 \mathrm{~dB}$ for the circuit with no sever.

Next, we consider cancellation of a 3IM with injection of the difference frequency. Theoretically, this situation is similar to second harmonic injection when the second harmonic is out of the linear gain bandwidth. Even though the difference frequency is out of the linear gain bandwidth, the second-order product of the difference frequency and the fundamental will grow at a rate equal to the fundamental frequency growth rate [17]. With sufficiently large difference frequency input power, cancellation of the 3IM can be obtained as shown in Fig. 13. Note that the required difference frequency input power relative to the fundamental $\left(P_{f_{2}-f_{1}}-P_{f_{1}}=17.4 \mathrm{~dB}\right)$ is similar to the required second harmonic injection power relative to the fundamental when the second harmonic is out of the linear gain bandwidth $\left(P_{2 f_{1}}-P_{f_{1}}=15.4 \mathrm{~dB}\right)$. Also note that the difference frequency can be used to cancel either 3IM frequency, but not both 3IMs simultaneously. However, simultaneous reduction of both 3IMs with difference frequency injection is likely possible.

There is some question as to whether difference frequency injection will work in practice. Since the wavelength of the difference frequency is much longer than the physical TWT, it is not clear as to whether the mode resulting from the product of the difference frequency and the fundamental frequency will be produced in the device. Ultimately, an experimental test of the idea will be needed to determine if it works.

4) Multiple Signal Injection: Second and third harmonic injection was shown in Section III-A3 to control the level of the

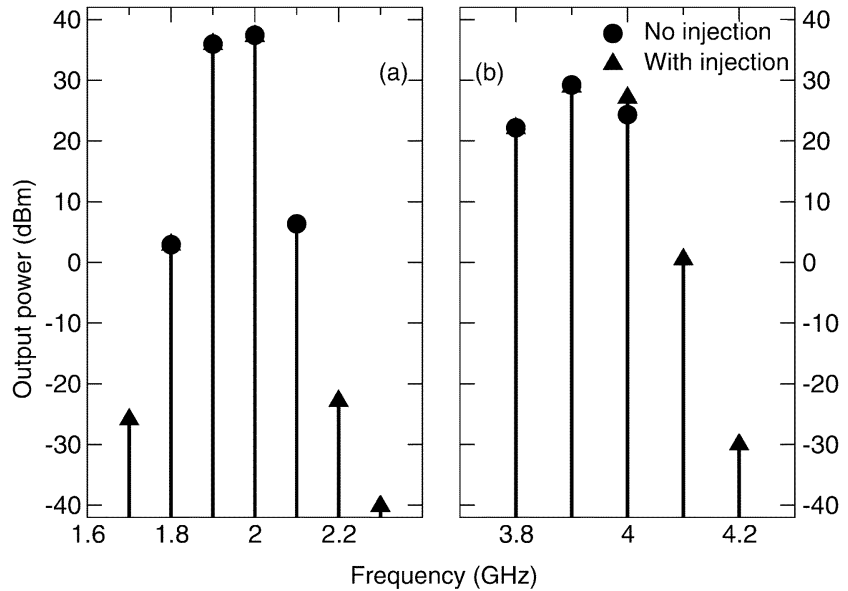

Fig. 14. Output spectrum (a) near fundamentals and (b) near second harmonics with and without 3IM and second harmonic injection. Note additional "intermodulation frequencies" due to the injection of the signals. Fundamental inputs $1.9,2.0 \mathrm{GHz}$ have input power $0.0 \mathrm{dBm}$ and respective phases of $0.0^{\circ}$ and $30.0^{\circ}$. Injected 3IM and second harmonic $2.1,4.0 \mathrm{GHz}$ have input powers and phases of -30.0 and $-22.0 \mathrm{dBm}$ and $-13.0^{\circ}$ and $35.0^{\circ}$.

second harmonic while eliminating the need for precise control of the injected signal phases. When using signal injection to control the intermodulation spectrum, there are many cases when one might wish to inject multiple signals. For example, second harmonic and intermodulation injection or second harmonic and difference frequency injection may be used to control the intermodulation level and eliminate the requirement of precise phase control of the injected signals. Furthermore, one could imagine that for multiple fundamental signals, second harmonic and intermodulation pairs or second harmonic and difference frequency pairs could be used to control the many intermodulation products. However, the effects of injecting many signals on the other spectral components and whether the resulting spectra are improved over the original spectra are not known. While we do not attempt to answer this question here, we give a few examples of multiple signal injection for two fundamental frequencies using LATTE simulations.

In Fig. 14, we show the output spectrum with and without injection of a 3IM $(2.1 \mathrm{GHz})$ and second harmonic $(4.0 \mathrm{GHz})$ to cancel the 3IM. The targeted 3IM frequency is totally canceled while additional spectral components are produced near the fundamentals and second harmonics. Since the new intermodulation products are more than $60 \mathrm{~dB}$ below the fundamentals amplitudes, they may not represent a problem as a practical matter. A determination of the importance of these new distortion products would need to be made in the context of linearity requirements for each specific application. As was the case in second and third harmonic injection to control the second harmonic (Section III-A3), this scheme eliminates the necessity of precise phase control of the injected signals. This is accomplished by producing two output phasors whose lengths can be controlled to cancel the undesired output phasor by adjusting the injected signal input powers (see Fig. 8). Implementation of this concept might simply require a passive predistortion equalizer with an added "leg" to generate second harmonic content along with the 3IM content that is conventionally generated with such circuits. 


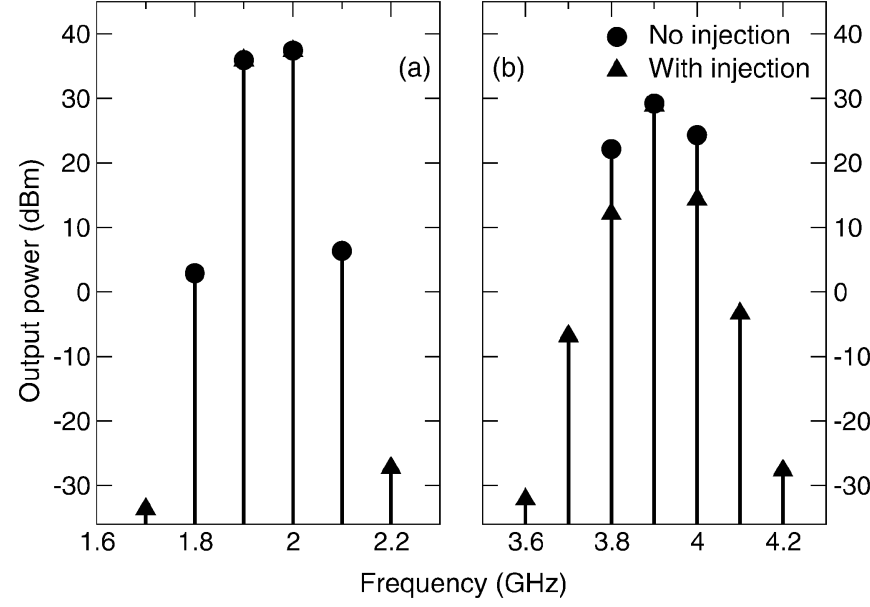

Fig. 15. Output spectrum (a) near fundamentals and (b) near second harmonics with and without second harmonic injection of both fundamentals. Note additional "intermodulation frequencies" due to the injection of the harmonic signals. Fundamental inputs $1.9,2.0 \mathrm{GHz}$ have input power $0.0 \mathrm{dBm}$ and respective phases of $0.0^{\circ}$ and $30.0^{\circ}$. Injected harmonics $3.8,4.0 \mathrm{GHz}$ have input powers and phases of -23.25 and $-19.4 \mathrm{dBm}$ and $66.5^{\circ}$ and $136.1^{\circ}$.

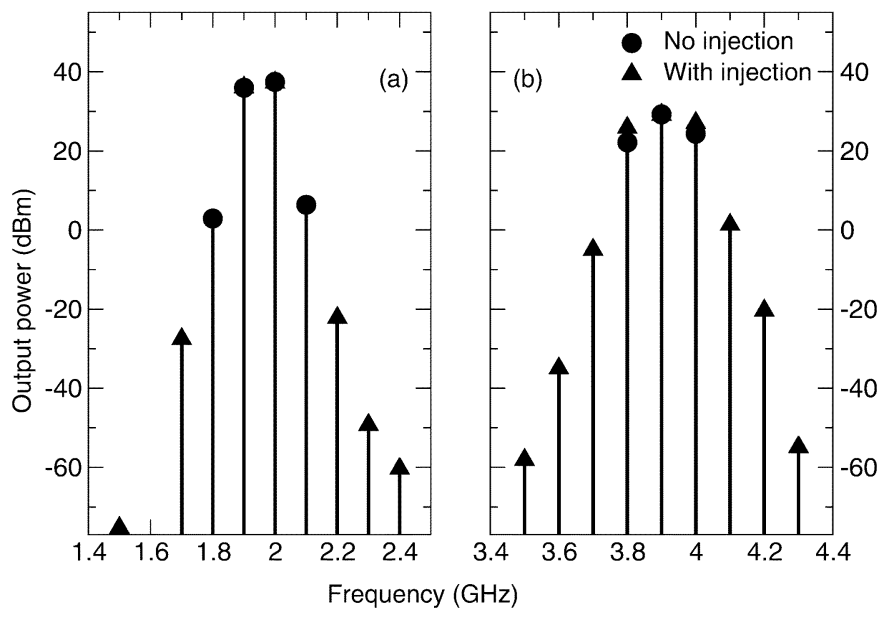

Fig. 16. Output spectrum (a) near fundamentals and (b) near second harmonics with and without injection of both 3IMs $(1.8,2.1 \mathrm{GHz})$ and both second harmonics $(3.8,4.0 \mathrm{GHz})$. Note additional "intermodulation frequencies" due to injection the of the signals. Fundamental inputs 1.9, 2.0 $\mathrm{GHz}$ have input power $0.0 \mathrm{dBm}$ and respective phases of $0.0^{\circ}$ and $30.0^{\circ}$. Injected 3IM and second harmonics 1.8, 2.1, 3.8, 4.0 GHz have input powers and phases of $-26.7,-30.0,-25.0$, and $-22.0 \mathrm{dBm}$ and $-150.0^{\circ},-13.0^{\circ}$, $-140.0^{\circ}$, and $35.0^{\circ}$. Large dynamic range of the figure is so that all of the spectral components are shown.

Next, we consider injecting the second harmonics of both of the fundamental frequencies. In Fig. 15, we show the output spectra with and without injection of both second harmonics. The original 3IM frequencies are totally canceled and there is some cancellation at the second harmonic as well, while some additional spectral components appear due to the additional input signals. The levels of the additional signals near the fundamentals are much lower than the original 3IM frequencies. There are several components generated near the second harmonics, although it is thought that these could be filtered and would be less of a concern than the spectrum near the fundamental.
Finally, in Fig. 16 we show results of injecting the 3IMs and second harmonics of both of the fundamental signals. The 3IM frequencies are canceled without a requirement of precise input phase control, but the number of additional spectral components becomes quite large. However, all of the signals near the fundamentals are substantially lower than the 3IMs without the injection. If one can tolerate a complicated spectrum near the second harmonics, it is thought that this scheme may be desirable.

We have done some testing of the above multiple signal injection schemes for fundamental inputs of $5 \mathrm{dBm}$, which start to show the onset of saturation effects in the intermodulation and harmonic signals. For second harmonic injection of both fundamentals, simultaneous cancellation of both 3IM frequencies was found to be possible. However, in the case of injecting both 3IMs and both second harmonics we were unable to find, with a coarse search, injection input values to cancel both of the 3IM frequencies simultaneously. The general existence of such inputs is a subject of future study.

\section{CONCLUSION}

We present an interpretation of harmonic injection and, more generally, "signal injection" in a TWT as the cancellation of "driven" and "nonlinear" modes. The interpretations are based on an approximate analytic solution to an approximate nonlinear model. Furthermore, we use "large signal" simulations to test whether the modal solution structure is valid in saturated conditions. In general, we find that the large signal simulations indeed show the abrupt phase change signature of the approximate analytic solutions.

The idea has existed for some time that the physical mechanism of harmonic injection is cancellation of such modes [4], [10]. However, we present the first quantitative theory to predict the existence of the modes. Furthermore, the quantitative theory provides further insights into harmonic injection. For example, a phasor picture provided by the theory demonstrates how multiple harmonic injection can eliminate the sensitive dependence of the output signals on the phase of the input signals. Also, the analytic solutions elucidate the many effects of the injected signals being outside of the linear gain bandwidth of the TWT and the role of circuit severs in these cases. Some experimentation is still required to verify all of the notions put forward in the paper.

\section{APPENDIX I}

\section{S-MUSE GENERAL ANALYTIC SOLUTION}

In this Appendix, we state the general analytic solution to the S-MUSE model, referring the reader to [12], [17] for the derivation. We use the vector notation of [11] where $\mathbf{x}_{\ell}=\left[\mathbf{x}_{\ell_{1}} \ldots \mathbf{x}_{\ell_{5}}\right]^{T}=\left[\tilde{V}_{\ell} \tilde{I}_{\ell} \tilde{E}_{\ell} \tilde{v}_{\ell} \tilde{\rho}_{\ell}\right]^{T}$. Due to the Fourier series used in the model, for each positive frequency $f_{\ell}$ there is a corresponding negative frequency indexed by $-\ell$ with $f_{-\ell}=-f_{\ell}$. Furthermore, $\mathbf{x}_{-\ell}=\mathbf{x}_{\ell}^{*}$. The differential equation for $\mathbf{x}_{\ell}$ is

$$
\dot{\mathbf{x}}_{\ell}=\mathbf{A}_{\ell} \mathbf{x}_{\ell}+\sum_{\substack{m, n \\ f_{m}+f_{n}=f_{\ell}}} \mathbf{H}_{\ell m n}\left(\mathbf{x}_{m}, \mathbf{x}_{n}\right)
$$


where matrix and tensor components $\mathbf{A}_{\ell_{i j}}$ and $\mathbf{H}_{\ell_{i} m_{j} n_{k}}$ are listed in [11, Appendix II]. One can show that (6) may be solved with a series solution

$$
\mathbf{x}_{\ell}=\sum_{\alpha=1}^{\infty} \mathbf{x}_{\ell}^{(\alpha)}
$$

and that this series converges under the appropriate conditions [12], [17]. The index $\alpha$ is related to the order of intermodulation product [17]. The formulas for the terms of the series are given by

$$
\begin{aligned}
\mathbf{x}_{\ell}^{(1)}= & e^{\mathbf{A}_{\ell} z} \mathbf{w}_{\ell} \quad \alpha=1 \\
\mathbf{x}_{\ell}^{(\alpha)}= & \int_{0}^{z} e^{\mathbf{A}_{\ell}(z-\tau)} \sum_{\beta=1}^{\alpha-1} \sum_{\substack{m, n \\
f_{m}+f_{n}=f_{\ell}}} \\
& \mathbf{H}_{\ell m n}\left(\mathbf{x}_{m}^{(\beta)}(\tau), \mathbf{x}_{n}^{(\alpha-\beta)}(\tau)\right) d \tau, \quad \alpha \geq 2
\end{aligned}
$$

where $\mathbf{w}_{\ell}$ contains the initial values for frequency $f_{\ell} \omega_{0}[11]$ and $e^{\mathbf{A}_{\ell} z}$ is the matrix exponential of the matrix $\mathbf{A}_{\ell} z$ [18]. The driven frequencies include the injected signals and each driven frequency has a nonzero component in $\mathbf{w}_{\ell}$.

Due to the recursive structure of (9), it may be written as

$$
\mathbf{x}_{\ell}^{(\alpha)}=\int_{0}^{z} e^{\mathbf{A}_{\ell}(z-\tau)} \sum_{q=1}^{M}\left(\sum_{r=0}^{N_{q}} \tau^{r} \mathbf{c}_{q}^{r}\right) e^{\sigma_{q} \tau} d \tau .
$$

In (10), a term of the forcing exponential, i.e., $\tau^{r} \mathbf{c}_{q}^{r} e^{\sigma_{q} \tau}$, is formed by the quadratic nonlinearity in (9) represented by $\mathbf{H}_{\ell m n}$. These terms arise from the mixing of driven frequencies (including injected signals) and, depending on the injection scheme, can correspond to the modes one wishes to cancel or the modes performing the cancellation. If the exponent $\sigma_{q}$ in the integral formula (10) is equal to an eigenvalue of $\mathbf{A}_{\ell}$, then powers of $z$ appear multiplying the exponentials in the result of the integral for $\mathbf{x}_{\ell}^{(\alpha)}$. The factor $\tau^{r}$ appears in (10) to account for the fact that $\mathbf{x}_{m}^{(\beta)}$ and $\mathbf{x}_{n}^{(\alpha-\beta)}$ may contain such powers of $z$. Cases when $\sigma_{q}$ is equal to an eigenvalue of $\mathbf{A}_{\ell}$ are referred to as "resonant forcing," and the modes that result involving powers of $z$ are referred to as "secular modes."

Modes are characterized by the arguments of their complex exponentials. The result of the integral in (10) has modes characterized by either $\sigma_{q}$ or the eigenvalues of $\mathbf{A}_{\ell}, \lambda_{\ell_{i}}, i=1, \ldots, 5$. Formulas for these modes are given as follows.

The $\lambda_{\ell_{k}}$ mode of (10) is

$$
\sum_{q=1}^{M} \sum_{r=0}^{N_{q}} \mathbf{P}_{\ell} \mathbf{R}_{\ell}\left(k, r, \sigma_{q}\right) \mathbf{P}_{\ell}^{-1} \mathbf{c}_{q}^{r} e^{\lambda_{\ell_{k}} z}, \quad k=1, \ldots, 5 .
$$

The $\sigma_{q}$ modes of (10) are as follows.

1) For $\sigma_{q} \neq \lambda_{\ell_{i}}, i=1, \ldots, 5$

$$
\sum_{r=0}^{N_{q}}\left[\sum_{k=0}^{N_{q}-r} \mathbf{P}_{\ell} \mathbf{S}_{\ell}\left(\sigma_{q}, r, k\right) \mathbf{P}_{\ell}^{-1} \mathbf{c}_{q}^{r+k}\right] z^{r} e^{\sigma_{q} z} .
$$

2) For $\sigma_{q}=\lambda_{\ell_{i}}$,

$$
\begin{aligned}
\mathbf{P}_{\ell}\left\{\mathbf{T}_{\ell}\left(N_{q}\right) \mathbf{P}_{\ell}^{-1} \mathbf{c}_{q}^{N_{q}} z^{N_{q}+1}\right. \\
\quad+\sum_{r=0}^{N_{q}}\left[H(r) \mathbf{T}_{\ell}(r-1) \mathbf{P}_{\ell}^{-1} \mathbf{c}_{q}^{r-1}\right. \\
\left.\left.\quad+\left(\sum_{k=0}^{N_{q}-r} \mathbf{S}_{\ell}\left(\sigma_{q}, r, k\right) \mathbf{P}_{\ell}^{-1} \mathbf{c}_{q}^{r+k}\right)\right] z^{r}\right\} e^{\sigma_{q} z} .
\end{aligned}
$$

In (11)-(13), $\mathbf{P}_{\ell}$ and $\mathbf{P}_{\ell}^{-1}$ are the modal matrix of $\mathbf{A}_{\ell}$ and its inverse, respectively [18]. The matrices $\mathbf{R}_{\ell}, \mathbf{S}_{\ell}$, and $\mathbf{T}_{\ell}$ are defined by the equations shown at the bottom of the page.

\section{APPENDIX II}

\section{FIRST-, SECOND-, AND THIRD-ORDER MOdE AMPLITUdES}

In this Appendix, we provide special cases of the formulas in Appendix I that are required to compute solutions of the dominant modes for the cases discussed in the text.

The complex exponential modes of the vector $\mathbf{x}_{\ell}^{(\alpha)}$ (see Appendix I) may be indexed by $p$

$$
\mathbf{x}_{\ell}^{(\alpha)}(z)=\sum_{p=1}^{N_{\ell}^{(\alpha)}} \mathbf{a}_{\ell}^{(\alpha)[p]} e^{\left(\mu_{\ell}^{(\alpha)[p]}+i \kappa_{\ell}^{(\alpha)[p]}\right) z}
$$

with $\mathbf{a}_{\ell}^{(\alpha)[p]}$ a complex vector and $\mu_{\ell}^{(\alpha)[p]}, \kappa_{\ell}^{(\alpha)[p]}$ real numbers. Sums like (14) are ordered so that $\mu_{\ell}^{(\alpha)[1]} \geq \mu_{\ell}^{(\alpha)[2]} \geq \cdots \geq$ $\mu_{\ell}^{(\alpha)\left[N_{\ell}^{(\alpha)}\right]}$. The cases in the text do not require secular modes so a factor of $z$ does not appear in (14).

To keep the formalism as simple as possible, we provide formulas only for the cases when the injected signals are in the linear gain bandwidth of the TWT. In these cases, the solutions for fixed $\alpha$ can be approximated by a single "dominant mode" in the case of single signal injection, or several "dominant modes" in the case of multisignal injection. The total solution (1) is then a sum of the dominant modes for the different values of $\alpha$.

When the injected signals are out of the linear gain bandwidth the number of modes required to represent the solutions

$$
\begin{aligned}
\mathbf{R}_{\ell_{i, j}}\left(k, r, \sigma_{q}\right) & \equiv \begin{cases}\frac{(-1)^{r+1} r !}{\left(\sigma_{q}-\lambda_{\ell_{k}}\right)^{r+1}} ; & i=j=k \text { and } \lambda_{\ell_{i}} \neq \sigma_{q} \\
0 ; & \text { otherwise }\end{cases} \\
\mathbf{S}_{\ell_{i, j}}\left(\sigma_{q}, r, k\right) & \equiv \begin{cases}(-1)^{k} \frac{(r+k) !}{r !} \frac{1}{\left(\sigma_{q}-\lambda_{\ell_{j}}\right)^{k+1}} ; & i=j \text { such that } \lambda_{\ell_{i}} \neq \sigma_{q} \\
0 ; & \text { otherwise }\end{cases} \\
\mathbf{T}_{\ell_{i, j}}(r) & \equiv \begin{cases}\frac{1}{r+1} ; & i=j \text { such that } \lambda_{\ell_{i}}=\sigma_{q} \text { and } r \neq-1 \\
0 ; & \text { otherwise. }\end{cases} \\
H(r) & = \begin{cases}1 & r \geq 1 \\
0 & r=0\end{cases}
\end{aligned}
$$


increases substantially, and the formulas for the solutions involve more caveats and technicalities. The general result allowing computation of all modes is given in Appendix I.

We give formulas for first-order (linear), second-order, and third-order products. The formulas may be used to compute all of the cases in the paper in which the injected signal is in the linear gain bandwidth of the TWT.

\section{A. First-Order (Linear) Solution}

The $p=1$ mode of the first-order $(\alpha=1)$ drive frequencies (including injected signals) is given by

$$
\mathbf{x}_{\ell}^{(1)[1]}=\mathbf{a}_{\ell}^{(1)[1]} e^{\mu_{\ell}^{(1)[1]} z} e^{i \kappa_{\ell}^{(1)[1]} z}
$$

where

$$
\begin{aligned}
\mathbf{a}_{\ell}^{(1)[1]} & =\mathbf{P}_{\ell} \mathbf{Q} \mathbf{P}_{\ell}^{-1} \mathbf{w}_{\ell} \\
\mu_{\ell}^{(1)[1]} & =\operatorname{Re}\left\{\lambda_{\ell_{1}}\right\} \\
\kappa_{\ell}^{(1)[1]} & =\operatorname{Im}\left\{\lambda_{\ell_{1}}\right\}
\end{aligned}
$$

and

$$
\mathbf{Q}_{i, j}= \begin{cases}1 & i=j=1 \\ 0 & \text { otherwise. }\end{cases}
$$

We assume that $\mathbf{P}_{\ell}$, the modal matrix of $\mathbf{A}_{\ell}$, has the eigenvector associated with $\lambda_{\ell_{1}}$, the eigenvalue of $\mathbf{A}_{\ell}$ with the largest real part, in its first column. Then, for (1) we have

$$
\begin{aligned}
A_{\ell}^{\mathrm{dr}} & =\mathbf{a}_{\ell_{1}}^{(1)[1]} \\
\mu_{\ell}^{\mathrm{dr}} & =\mu_{\ell}^{(1)[1]} \\
\kappa_{\ell}^{\mathrm{dr}} & =\kappa_{\ell}^{(1)[1]} .
\end{aligned}
$$

For each frequency $f_{\ell} \omega_{0}$ with a nonzero input power there is an equation (1) with a driven mode solution given by (20)-(22).

\section{B. Second-Order Solution}

Next, we solve for second-order products $(\alpha=2)$ of the injected signals. We give formulas for one mode in the sum over $q$ in (1) and note that the number of required modes and ordering of the modes is application dependent. A second-order product has associated with it a pair of indexes $m$ and $n$ such that $f_{m}+f_{n}=f_{\ell}$. Extracting only the dominant mode from (12) of Appendix I we have

$$
\mathbf{x}_{\ell}^{(2)[q]}=\mathbf{a}_{\ell}^{(2)[q]} e^{\mu_{\ell}^{(2)[q]} z} e^{i \kappa_{\ell}^{(2)[q]} z}
$$

(24)-(27), as shown at the bottom of the page, and we have assumed that $\mu_{m}^{(1)[1]}+\mu_{n}^{(1)[1]}>\operatorname{Re}\left\{\lambda_{\ell_{1}}\right\}$. The eigenvalues $\lambda_{\ell_{j}}$ of $\mathbf{A}_{\ell}$ have the same ordering as the eigenvectors of $\mathbf{A}_{\ell}$ appearing in columns of $\mathbf{P}_{\ell}$. The matrix $\mathbf{S}_{\ell}$ is given in Appendix I.
Then, for a mode of (1), we have

$$
\begin{aligned}
A_{\ell}^{\mathrm{nl}[q]} & =\mathbf{a}_{\ell_{1}}^{(2)[q]} \\
\mu_{\ell}^{\mathrm{nl}[q]} & =\mu_{\ell}^{(2)[q]} \\
\kappa_{\ell}^{\mathrm{nl}[q]} & =\kappa_{\ell}^{(2)[q]} .
\end{aligned}
$$

This mode can describe sum and difference frequencies of the injected signals.

\section{Third-Order Solution}

Lastly, we solve for third-order products $(\alpha=3)$ of the injected signals. Given the dominant mode of the injected signal $\mathbf{x}_{m}^{(1)[1]}$ from (15) and a dominant mode of a second-order product $\mathbf{x}_{n}^{(2)[1]}$ from (23), one has

$$
\mathbf{x}_{\ell}^{(3)[1]}=\mathbf{a}_{\ell}^{(3)[1]} e^{\mu_{\ell}^{(3)[1]} z} e^{i \kappa_{\ell}^{(3)[1]} z}
$$

where

$$
\begin{aligned}
\mathbf{a}_{\ell}^{(3)[1]}= & \mathbf{P}_{\ell} \mathbf{S}_{\ell}\left(\mu_{\ell}^{(3)[1]}+i \kappa_{\ell}^{(3)[1]}, 0,0\right) \mathbf{P}_{\ell}^{-1} \mathbf{c}_{\ell}^{(3)[1]} \\
\mathbf{c}_{\ell}^{(3)[1]}= & \mathbf{H}_{\ell m n}\left(\mathbf{a}_{m}^{(1)[1]}, \mathbf{a}_{n}^{(2)[1]}\right) \\
& +\mathbf{H}_{\ell n m}\left(\mathbf{a}_{n}^{(2)[1]}, \mathbf{a}_{m}^{(1)[1]}\right) \\
\mu_{\ell}^{(3)[1]}= & \mu_{m}^{(1)[1]}+\mu_{n}^{(2)[1]} \\
\kappa_{\ell}^{(3)[1]}= & \kappa_{m}^{(1)[1]}+\kappa_{n}^{(2)[1]} .
\end{aligned}
$$

Therefore, for (1), we have for the dominant mode of the thirdorder product

$$
\begin{aligned}
A_{\ell}^{\mathrm{nl}[1]} & =\mathbf{a}_{\ell_{1}}^{(3)[1]} \\
\mu_{\ell}^{\mathrm{nl}[1]} & =\mu_{\ell}^{(3)[1]} \\
\kappa_{\ell}^{\mathrm{nl}[1]} & =\kappa_{\ell}^{(3)[1]} .
\end{aligned}
$$

Unlike the $\alpha=2$ case, we allow for only one third-order mode in the total solution (1). Although a more general formalism is possible, the examples provided in the paper do not require it.

\section{ACKNOWLEDGMENT}

The authors would like to thank A. Singh for useful feedback on the manuscript.

\section{REFERENCES}

[1] D. Jennings, A. Bateman, and J. McGeehan, "Adjacent channel power and error-vector magnitude performance of reduced complexity CALLUM systems," IEE Proc.-Commun., vol. 146, no. 5, pp. 297-302, Oct. 1999.

[2] J. Hamilton and D. Zavadil, "Harmonically-enhanced two-octave TWTA," Microwave J., vol. 15, pp. 24-25, 1972.

[3] O. Sauseng, M. Huisjen, and W. Garrigus, "Reduction of intermodulation distortion with harmonic injection for wideband travelling-wave tubes," IEDM Tech. Dig., pp. 411-414, 1975.

$$
\begin{aligned}
\mathbf{a}_{\ell}^{(2)[q]} & =\mathbf{P}_{\ell} \mathbf{S}_{\ell}\left(\mu_{\ell}^{(2)[q]}+i \kappa_{\ell}^{(2)[q]}, 0,0\right) \mathbf{P}_{\ell}^{-1} \mathbf{c}_{\ell}^{(2)[q]} \\
\mathbf{c}_{\ell}^{(2)[q]} & =\left\{\begin{array}{lc}
\mathbf{H}_{\ell m n}\left(\mathbf{a}_{m}^{(1)[1]}, \mathbf{a}_{n}^{(1)[1]}\right) \\
\mathbf{H}_{\ell m n}\left(\mathbf{a}_{m}^{(1)[1]}, \mathbf{a}_{n}^{(1)[1]}\right)+\mathbf{H}_{\ell n m}\left(\mathbf{a}_{n}^{(1)[1]}, \mathbf{a}_{m}^{(1)[1]}\right) & \begin{array}{c}
m+f_{n}=f_{\ell} \\
m \neq n
\end{array} \\
f_{m}+f_{n}=f_{\ell}
\end{array}\right. \\
\mu_{\ell}^{(2)[q]} & =\mu_{m}^{(1)[1]}+\mu_{n}^{(1)[1]} \\
\kappa_{\ell}^{(2)[q]} & =\kappa_{m}^{(1)[1]}+\kappa_{n}^{(1)[1]}
\end{aligned}
$$


[4] W. Garrigus and M. Glick, "Multi-octave high-power TWT operation," Microwave J., vol. 18, pp. 35-40, 1975.

[5] M. Wirth, A. Singh, J. Scharer, and J. Booske, "Third-order intermodulation reduction by harmonic injection in a TWT amplifier," IEEE Trans. Electron Devices, vol. 49, pp. 1082-1084, 2002.

[6] A. Singh, J. Wöhlbier, J. Booske, and J. Scharer, "Experimental verification of the mechanisms for nonlinear harmonic growth and suppression by harmonic injection in a traveling wave tube," Phys. Rev. Lett., vol. 92, 2004, submitted for publication.

[7] N. Dionne, "Harmonic generation in octave bandwidth traveling-wave tubes," IEEE Trans. Electron Devices, vol. ED-17, pp. 365-372, 1970.

[8] S. Datta, P. Jain, M. R. Narayan, and B. Basu, "Eulerian analysis for harmonic generation and its control in a helix travelling-wave tube," Int. J. Electron., vol. 85, no. 3, pp. 377-395, 1998.

[9] S. Datta, P. Jain, and B. Basu, "Control of IM3 distortion in helix TWT's by harmonic injection-An Eulerian hydrodynamical study," IEEE Trans. Electron Devices, vol. 48, pp. 62-66, Jan. 2001.

[10] J. Mendel, "Helix and coupled-cavity travelling-wave tubes," Proc IEEE, vol. 61, pp. 280-288, 1973.

[11] J. Wöhlbier, J. Booske, and I. Dobson, "The multifrequency spectral Eulerian (MUSE) model of a traveling wave tube," IEEE Trans. Plasma Sci., vol. 30, pp. 1063-1075, 2002.

[12] J. Wöhlbier, "Nonlinear distortion and suppression in traveling wave tubes: insights and methods," Ph.D. dissertation, Univ. Wisconsin-Madison, 2003.

[13] J. Pierce, Traveling Wave Tubes. Princeton, NJ: Van Nostrand, 1950.

[14] J. Plouin, "Origins of saturation in a traveling wave tube," in Proc. Third IEEE Int. Vacuum Electronics Conf., 2002, pp. 318-319.

[15] C. Fan and K. Cheng, "Amplifier linearization using simultaneous harmonic and baseband injection," IEEE Microwave Wireless Components Lett., vol. 11, no. 10, pp. 404-406, Oct. 2001.

[16] A. Singh, J. Wöhlbier, J. Scharer, and J. Booske, "Injection schemes for TWT linearization," in Proc. IVEC 2003 Conf., 2003.

[17] J. Wöhlbier, I. Dobson, and J. Booske, "Generation and growth rates of nonlinear distortions in a traveling wave tube," Phys. Rev. E, vol. 66, 2002.

[18] C. Chen, Linear System Theory and Design, 2nd ed. New York: Oxford Univ. Press, 1984

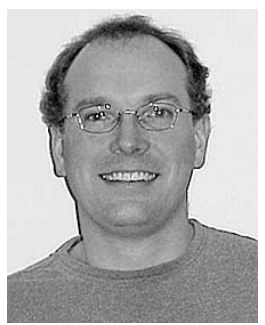

John G. Wöhlbier (M'03) received the Ph.D. degree in electrical engineering from the University of Wisconsin, Madison, in 2003.

In the summer of 2003, he was an engineering physics Postdoctoral Scientist at the University of Wisconsin, working on ballooning instabilities in three-dimensional plasmas. Presently, he is at Los Alamos National Laboratory, Los Alamos, NM, on a National Security Postdoctoral Fellowship. His research interests include microwave vacuum electronics, plasma physics, fluid dynamics, dynamica systems, and applied math

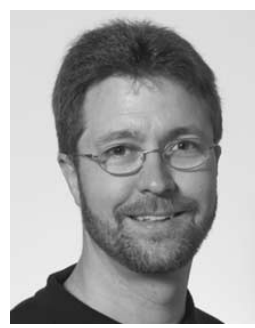

John H. Booske (S'82-M'85-SM'93) received the $\mathrm{Ph} . D$. degree in nuclear engineering from the University of Michigan, Ann Arbor, in 1985.

From 1985 to 1989 , he was a Research Scientist at the University of Maryland, College Park. In 1990, he joined the faculty of the University of Wisconsin, Madison, where he is a Professor of Electrical an Computer Engineering. In 2001, he was appointed Director of the University of Wisconsin's Materials Science Program. His research interests include the experimental and theoretical study of coherent electromagnetic radiation sources and their applications, with emphasis in the RF, microwave, millimeter-wave, and terahertz regimes. Recent research activities include vacuum electronics, microwave and RF heating of materials, biological/biomedical applications of microwave and RF fields, high current density field emission cathodes, and ultrashallow junction fabrication in silicon CMOS transistors.

Prof. Booske received the University of Wisconsin Vilas Associate Award for research excellence and the U.S. National Science Foundation Presidential Young Investigator Award. He has been honored with many teaching awards, including the University of Wisconsin Chancellor's Distinguished Teaching Award and the Benjamin Smith Reynolds Award for Excellence in Teaching Engineering. He served as Codirector of a U.S. Department of Defense MURI99 consortium on innovative microwave vacuum electronics and as Director of a MURI04 consortium on cathode field emission and RF window breakdown in high-power microwave sources.

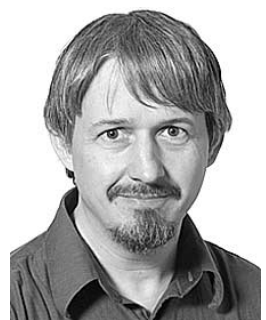

Ian Dobson (M'89-SM'98) received the B.A. degree in mathematics from Cambridge University, Cambridge, U.K., in 1978 and the Ph.D. degree in electrical engineering from Cornell University, Ithaca, NY, in 1989

He worked from 1978 to 1983 as a Systems Analyst for the British firm EASAMS, Ltd. In 1989, he joined the University of Wisconsin, Madison, where he is now a Professor in electrical and computer engineering. His current interests are applications of nonlinear dynamics, electric power system instabilities, power electronics, and self-organized criticality. 